\title{
Expression of transcription factors in MEN1- associated pancreatic neuroendocrine tumors
}

\author{
Yasutaka Takeda, Yukihiro Fujita, Kentaro Sakai, Tomoe Abe, Tomonobu Nakamura, \\ Tsuyoshi Yanagimachi, Hidemitsu Sakagami, Jun Honjo, Atsuko Abiko, Yuichi Makino \\ and Masakazu Haneda
}

Division of Metabolism and Biosystemic Science, Department of Internal Medicine, Asahikawa Medical University, Asahikawa, Japan
Correspondence should be addressed to Y Fujita Email

yfujita@asahikawa-med.ac.jp

\section{Summary}

MEN1-associated pancreatic neuroendocrine tumors (pNETs) may potentially express distinct hormones, but the mechanism has not been elucidated. Transcription factors such as MafA and Pdx1 have been identified to lead to beta cell differentiation, while Arx and Brn4 to alpha cell differentiation in developing pancreas. We hypothesized those transcription factors are important to produce specific hormones in pNETs, similarly to developing pancreas, and examined the expression of transcription factors in a case of MEN1 who showed immunohistological coexistence of several hormone-producing pNETs including insulinoma. A 70-year-old woman was found to manifest hypoglycemia with non-suppressed insulinemia and hypercalcemia with elevated PTH level. She was diagnosed as MEN1 based on the manifestation of primary hyperparathyroidism, pituitary adenoma and insulinoma, with genetic variation of MEN1 gene. She had pylorus-preserving pancreaticoduodenectomy because CT scan and SACl test indicated that insulinoma was localized in the head of the pancreas. Histopathological finding was MEN1-associated NET, G1. Interestingly, immunohistological examination of the resected pancreas revealed that two insulinomas, a glucagon-positive NET and a multiple hormone-positive NET coexisted. Hence, we examined the expression of transcription factors immunohistochemically to elucidate the role of the transcription factors in MEN1-associated hormone-producing pNETs. We observed homogeneous expressions of MafA and Pdx1 in insulinomas and Arx in glucagon-positive NET, respectively. Moreover, multiple hormone-positive NETs expressed several transcription factors heterogeneously. Collectively, our results suggested that transcription factors could play important roles in the production of specific hormones in MEN1-associated pNETs, similar to islet differentiation.

\section{Learning points:}

- To date, it has been shown that different hormone-producing tumors coexist in MEN1-associated pNETs; however, the underlying mechanism of the hormone production in MEN1-associated PNETs has not been well elucidated.

- Although this case presented symptomatic hypoglycemia, several hormone-producing pNETs other than insulinoma also coexisted in the pancreas.

- Immunohistochemical analysis showed MafA and Pdx1 expressions distinctly in insulinoma, and Arx expression particularly in a glucagon-positive NET, while a multiple hormone-positive NET expressed MafA, Pdx1 and Arx.

- Collectively, clinicians should consider that several hormone-producing PNETs may coexist in a MEN1 case and examine both endocrinological and histopathological analysis of PNETs, regardless of whether symptoms related to the excess of hormones are observed or not. 


\section{Background}

Multiple endocrine neoplasia (MEN) is characterized by the occurrence of tumors involving two or more endocrine glands within a single patient. MEN type 1 (MEN1) is characterized by the tumorigenesis in the parathyroid glands, the pancreatic islets and the anterior pituitary usually associated with genetic variation of MEN1 gene on long arm of chromosome 11 (11q13) (1). It has been shown that different hormone-producing tumors occasionally coexist in MEN1-associated pancreatic neuroendocrine tumors (pNETs) (2). However, the underlying mechanism still remains to be elucidated on how MEN1-associated pNETs can produce specific hormones.

In the developing pancreas, several specific transcription factors have been identified and play crucial roles in the islet cell differentiation and production of distinct hormones. Aristaless-related homeobox (Arx) and Brain-4 (Brn4) are involved in alpha cell differentiation and induce glucagon secretion, while MafA (v-MAF musculoaponeurotic fibrosarcoma oncogene homologue A) and Pdx1 (pancreatic and duodenal homeobox factor-1) are important for beta cell differentiation and production of insulin (3).

Here, we hypothesized that the expression of transcription factors involved in the differentiation of islet endocrine cells is essential to produce specific hormones similarly in pNETs. We reported a case of MEN1, which unveiled the relationship between the expression of transcription factors and production of pancreatic hormones in MEN1-associated pNETs.

\section{Case presentation}

A 70-year-old Japanese woman was found to be symptomatic hypoglycemia $(51 \mathrm{mg} / \mathrm{dL}$, normal range (NR): $70-109 \mathrm{mg} / \mathrm{dL}$ ) with non-suppressed insulinemia (immunoreactive insulin: $23.5 \mu \mathrm{U} / \mathrm{mL}, \quad 5.0-10.0 \mu \mathrm{U} /$ $\mathrm{mL}$, C-peptide immunoreactivity: $2.52 \mathrm{ng} / \mathrm{mL}$, NR: $1.40-4.40 \mathrm{ng} / \mathrm{mL}$ ). She also presented asymptomatic hypercalcemia and hypophosphatemia with elevated intact parathyroid hormone (PTH) level. A preliminary dynamic computed tomography (CT) scan showed an $8 \mathrm{~mm}$ enhanced nodule in the head of the pancreas during the early phase. She was previously diagnosed with osteoporosis and rheumatoid arthritis with history of bilateral knee joint replacement, but neither family history of MEN nor previous usage of glucose-lowering agents. She was $149.4 \mathrm{~cm}$ tall with a body weight of $60.4 \mathrm{~kg}$; her BMI was $27.1 \mathrm{~kg} / \mathrm{m}^{2}$. Other than mild obesity, she showed no abnormal findings on physical examination. The patient was referred to our department for further examinations.

\section{Investigation}

We conducted prolonged (up to $72 \mathrm{~h}$ ) supervised fasting test to confirm hypoglycemia accompanied with non-suppressed insulin secretion. After 36-h fast, she manifested significant hypoglycemia (plasma glucose: $41 \mathrm{mg} / \mathrm{dL}$ ) accompanied with non-suppressed insulin secretion (immunoreactive insulin: $14.79 \mu \mathrm{U} / \mathrm{mL}$, C-peptide immunoreactivity: $2.52 \mathrm{ng} / \mathrm{mL}$, proinsulin immunoreactivity: $26.5 \mathrm{pmol} / \mathrm{L})$. Thus, we diagnosed this case as insulinoma (4). Dynamic contrast-enhanced abdominal CT showed an 8-mm enhanced nodule in the head of the pancreas during the early phase (Fig. 1), similar to the previous study. Next, selective arterial calcium injection (SACI) test was conducted to examine the localization of insulinoma. Calcium-induced insulin secretion was observed in the gastroduodenal artery and the superior mesenteric artery. These results indicated insulinoma was localized in the head of the pancreas. We further performed endoscopic ultrasonography (EUS) to detect other micro-lesions, in addition to CT. EUS showed $12.2-\mathrm{mm}$ and $7.8-\mathrm{mm}$ round low echoic nodules in the head of the pancreas, which were compatible with pNETs. We also observed hypercalcemia (adjusted serum calcium: $11.8 \mathrm{mg} / \mathrm{dL}$, NR: $8.5-10.2 \mathrm{mg} / \mathrm{dL})$, hypophosphatemia (2.1 mg/dL, NR: $2.4-4.3 \mathrm{mg} / \mathrm{dL})$ and elevated urinary calcium excretion (FECa: 1.14\%). Endocrinological examinations showed elevated intact-PTH level (131.0pg/

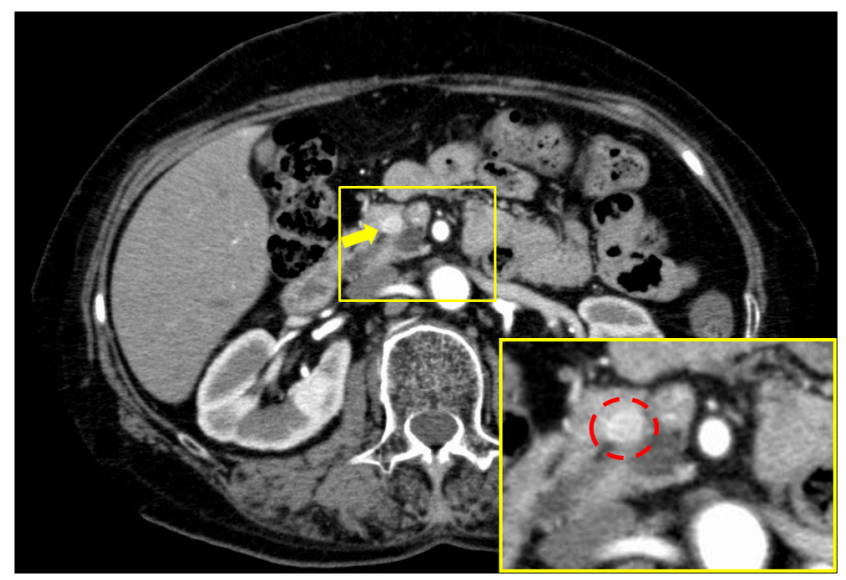

Figure 1

Contrast-enhanced computed tomography of pancreas revealed an $8 \mathrm{~mm}$ enhanced nodule of the head of the pancreas during early phase. 
A
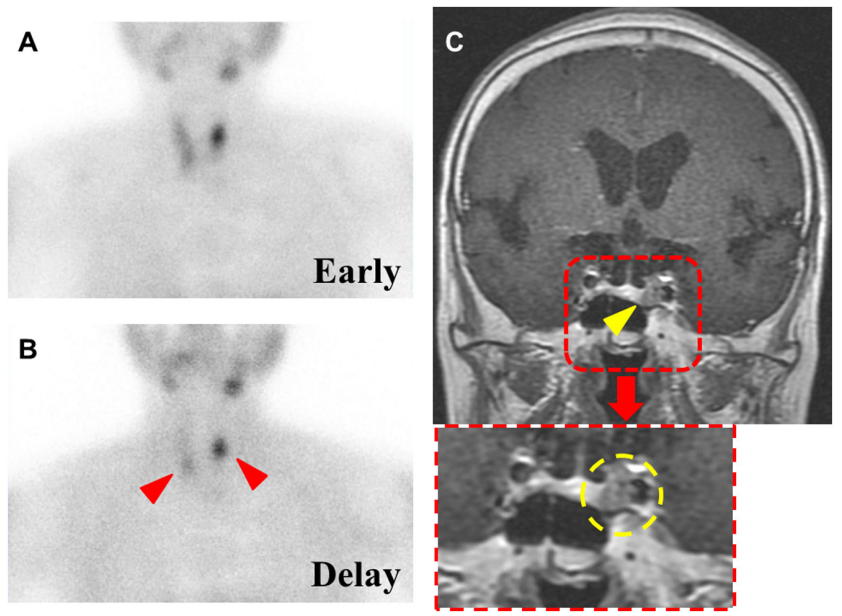

Figure 2

Images of the parathyroid glands and pituitary. Tc-99m sestamibi/ single-photon emission computed tomography (SPECT) scanning of parathyroid glands in early phase (A) and delayed phase (B). (C) Contrast-enhanced MRI of the pituitary showed a $10-\mathrm{mm}$ poorly enhanced nodule indicating adenoma.

mL, NR: 10-65 pg/mL) and elevated 1,25-dihydroxyvitamin D level (65 pg/mL, NR: 20-60 pg/mL) and elevated serum prolactin level $(428.2 \mathrm{ng} / \mathrm{mL}$, postmenopausal women NR: 3.1-15.4 ng/mL). Neck ultrasonography indicated low echoic lesions at the dorsal side of the right lobe of thyroid gland. Additionally, Tc-99m sestamibi/single-photon emission computed tomography (SPECT) scanning showed the localized accumulation at parathyroid glands in the delayed phase (Fig. 2A and B). These findings led to a diagnosis of primary hyperparathyroidism (pHPT). Contrast-enhanced pituitary MRI showed a $10 \mathrm{~mm}$ poorly enhanced nodular lesion indicating a pituitary adenoma (Fig. 2C), although her anterior pituitary function test was normal except mild hyperprolactinemia without any compression symptoms. Collectively, she was clinically diagnosed as MEN1. Furthermore, a base substitution in exon 8 of MEN1 gene was observed (exon 8 c.376T $>$ G) by genome sequencing.

\section{Treatment}

She had pylorus-preserving pancreaticoduodenectomy (PPPD) for insulinoma. Histopathological finding was NET G1 (Ki67 index <1\%) of the pancreas. TNM staging was T1N0M0, Stage IA (AJCC/UICC). Interestingly, several hormone-producing pNETs coexisted in the resected pancreas, although this case presented symptomatic hypoglycemia with non-suppressed insulinemia.

\section{Immunohistochemical analysis}

We hypothesized the expression of transcription factors involved in the differentiation of islet endocrine cells is closely related to the production of specific hormones in pNETs, and examined the immunohistochemical expression of the islet hormones, Pdx1, MafA and Arx among four pNETs (pNET\#1-4, Fig. 3A, B, C and D) obtained from resected pancreas in this case. Embedded tissue was sliced into $3 \mu \mathrm{m}$-thick sections and deparaffinized with a series of xylene and ethanol. We performed antigen retrieval with sodium citrate buffer followed by microwave heating and treated sections with a protein-blocking reagent (Dako Japan Inc, Tokyo, Japan). We incubated slides with primary antibody overnight at $4^{\circ} \mathrm{C}$ and after washing incubated with secondary antibody for $2 \mathrm{~h}$ at room temperature in shading. Slides were washed and finally mounted in aqueous media (VECTASHIELD Mounting Medium; Vector Laboratories). Stained sections were observed under a fluorescence microscopy (BZ-8100; Keyence, Osaka, Japan). Other sections were also immunostained using an avidin-biotin complex immunoperoxidase method with 3.3'-diaminobenzidine after antigen retrieval, and treatment with hydrogen peroxide.

Antibodies were the following: guinea pig anti-insulin antibody (Millipore), mouse anti-glucagon antibody (Sigma-Aldrich), rabbit anti-somatostatin antibody (Yanaihara Institute Inc, Fujinomiya, Japan), rabbit anti-chromogranin A (CgA) antibody (Abcam), rabbit anti-MafA antibody ChIP grade (Abcam), rabbit antiPdx1 antibody (Millipore) and sheep anti-Arx antibody (R\&D Systems) as primary antibodies. AMCA anti-guinea pig IgG (Jackson ImmunoResearch), Alexa Fluor 488 antirabbit IgG, Alexa Fluor 594 anti-mouse IgG (Invitrogen), biotinylated anti-rabbit IgG antibody and biotinylated anti-sheep IgG antibody (Vector Laboratories, Burlingame, CA, USA) were used as secondary antibodies.

\section{Results: immunohistochemical analysis}

All pNETs were positive for CgA (Fig. 4C, I, O and U), supporting NETs. pNET\#1 (5 mm in diameter) and pNET\#2 (13 $\mathrm{mm}$ in diameter) were positive for only insulin (Fig. 4B and $\mathrm{H}$ ), so these were regarded as insulinoma. pNET\#1 and pNET\#2 showed both MafA and Pdx1 expressions homogeneously, while Arx expression was not observed (Fig. 4D, E, F, J, K and L). Contrarily, 

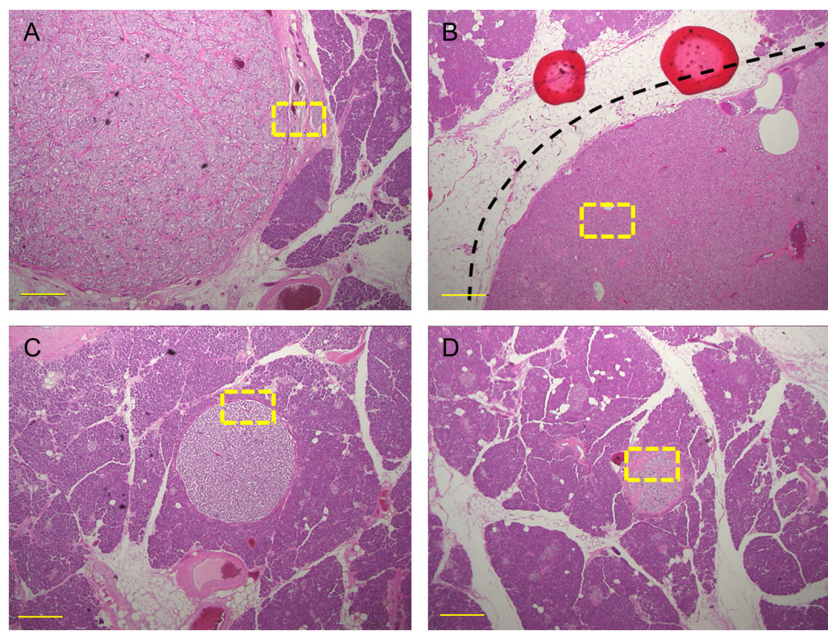

Figure 3

Pancreatic sections stained with hematoxylin-eosin. (A) pNET\#1 with a diameter of $5 \mathrm{~mm}$. (B) pNET\#2 with a diameter of $13 \mathrm{~mm}$. (C) pNET\#3 with a diameter of $1.5 \mathrm{~mm}$. (D) pNET\#4 with a diameter of $1 \mathrm{~mm}$. Bar $=500 \mu \mathrm{m}$. Magnification: $\times 80$.

pNET\#3 (1.5 $\mathrm{mm}$ in diameter) was positive for only glucagon (Fig. 4N). This pNET\#3 showed homogeneous Arx expression, while MafA and Pdx1 expressions were not observed (Fig. 4P, Q and R). Intriguingly, pNET\#4 (1 $\mathrm{mm}$ in diameter) was heterogeneously and sparsely stained with insulin, glucagon, and somatostatin (Fig. 4T). This multiple hormone-positive pNET\#4 showed co-expression of MafA, Pdx1 and Arx (Fig. 4V, $\mathrm{W}$ and $\mathrm{X}$ ). These results suggested that the expression of intrinsic transcription factors is essential to produce specific hormones in MEN1-associated pNETs, similar to islet differentiation.

\section{Outcome and follow-up}

Immediately after PPPD, plasma glucose levels were normalized and no more hypoglycemic symptom was noticed. Subsequently, she had parathyroidectomy for pHPT 3 months after PPPD and then vitamin D replacement therapy with alfacalcidol was initiated. Thereafter, her serum calcium and phosphate levels were maintained within normal range. Annual pituitary MRI showed no enlargement of pituitary adenoma up to the present time.

\section{Discussion}

To date, various transcription factors associated with the pancreatic development have been identified. In particular, it has been shown that the intrinsic expressions of transcription factors, such as Arx and Brn4 in alpha cell, and MafA and Pdx1 in beta cell, are essential to produce the specific hormones in islet differentiation (3). However, the underlying mechanism has not been elucidated on how MEN1-associated pNETs can produce specific pancreatic hormones. Hence, we hypothesized the expression of transcription factors involved in the islet endocrine differentiation might also be crucial and important to produce specific hormones in pNETs, and investigated the expression of transcription factors by immunohistochemical analysis.

In the present report, we showed coexistence of several distinct types of pNETs other than insulinoma, although this case presented hypoglycemic manifestation attributed to insulinoma. Here, we unveiled the possible relationship between the transcription factors and hormone production in MEN1-associated pNETs. First, our findings raise questions why different functionalized pNETs coexist in the pancreas of the single case similar to previous reports (2). As far as we searched, none of previous literatures gave us confident answers why several pNETs coexist in one MEN1 case. However, some previous studies can provide us important insights to speculate the possibility. Primarily, mutation of the MEN1 gene, a tumor suppressor gene, is generally observed both in sporadic and hereditary cases of MEN1. This gene mutation leads to loss of function of its protein product menin, causing tumorigenesis in MEN1 cases (1). Menin interacts with a number of proteins that are involved in transcriptional regulation, genome stability, cell division and proliferation. We assume that loss of function of menin could be a fundamental cause of coincidence of several pNETs in single MEN1 cases including our current case with a base substitution in exon 8 of MEN1 gene.

The basic helix-loop-helix transcription factor Neurogenin 3 (Ngn3) is indispensable for the early development of pancreatic endocrine cells and essential for the downstream differentiation in rodent $(5,6)$ and human (7). In addition, menin is also essential for development of the pancreatic endocrine cells and especially crucial for formation of Ngn3 expressing endocrine progenitor cells (8). These observations provide us an inference that dysgenesis of Ngn3-expressing cells, attributed to menin disruption by MEN1 gene variations, may lead functionally divergent coincidence of pNETs in MEN1 patients including our case. Furthermore, as a new insight, Lu et al. unveiled that menin regulates the 


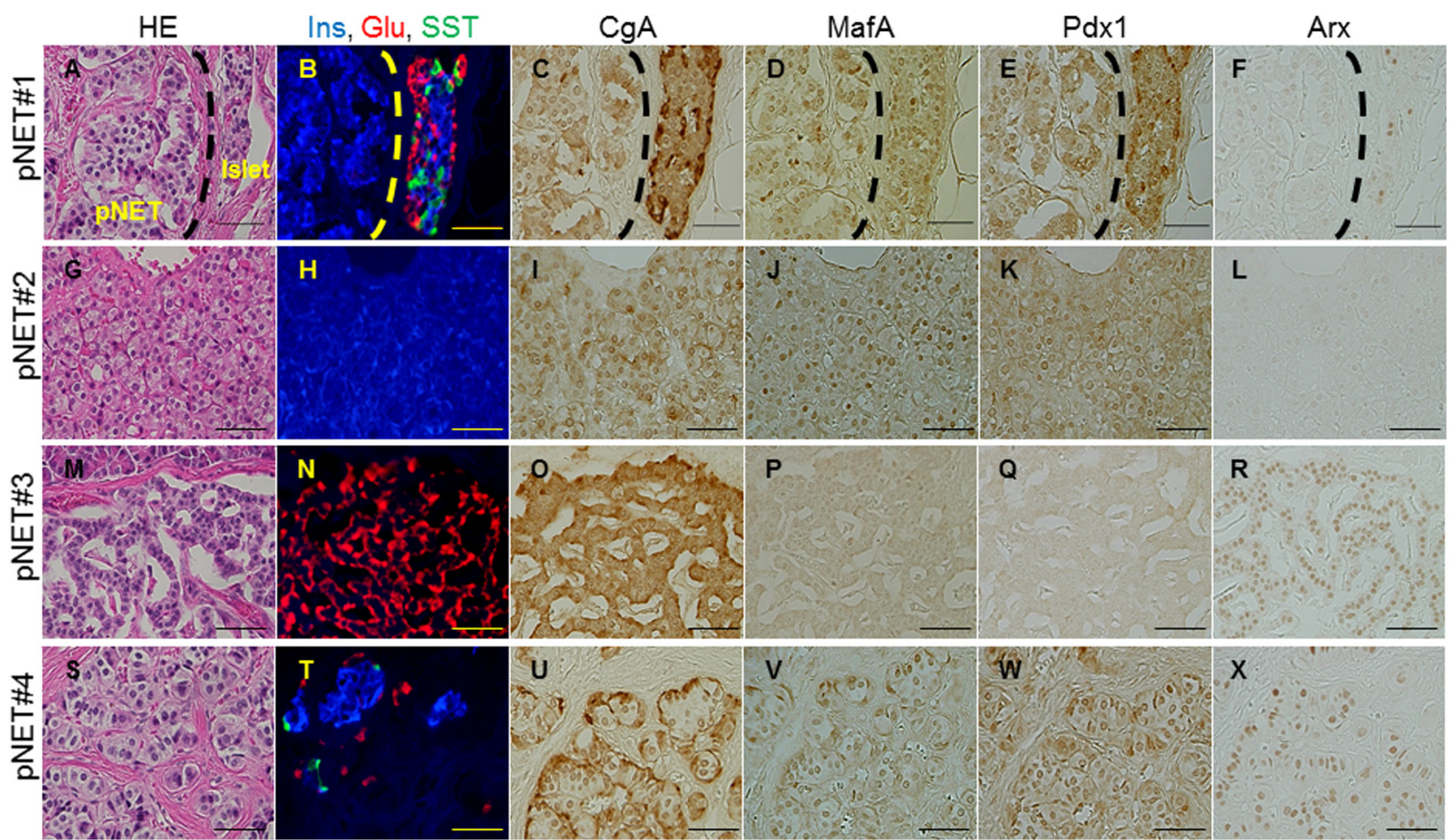

Figure 4

Immunohistochemical analysis of pNET\#1 (A, B, C, D, E and F), pNET\#2 (G, H, I, J, K and L), pNET\#3 (M, N, O, P, Q and R) and pNET\#4 (S, T, U, V, W and X). ( $A, G, M$ and S) Hematoxylin-eosin staining. ( $B, H, N$ and $T$ ) Insulin (blue), glucagon (red) and somatostatin (green) immunostaining. (C, I, O and $U$ ) Chromogranin A (brown) immunostaining. (D, J, P and V) MafA (brown) immunostaining. (E, K, Q and W) Pdx1 (brown) immunostaining. (F, L, R and X) Arx (brown) immunostaining. Bar $=50 \mu \mathrm{m}$. Magnification: $\times 800$.

plasticity of pancreatic alpha cells, and deficit of menin can cause the transdifferentiation of alpha cells and subsequent multiple tumorigenesis of islet endocrine cells in Men1 mutant mice (9). We presume that functional loss of menin is likely to be an important mechanism underlying multiple pNETs development in MEN1 cases, similarly to the islet tumorigenesis in genetic ablation of menin in the mutated mice.

In the current study, we particularly focused on expression of MafA, Pdx1 and Arx in the hormone production of MEN1-associated pNETs. We demonstrated for the first time that the expression of intrinsic transcription factors can be essential to produce specific hormones in MEN1-associated pNETs, similar to islet endocrine differentiation. $P d x 1$ is a key transcription factor involved in pancreatic development, beta cell differentiation, and maintenance of the mature beta cell. MafA also plays an important role in maintenance of beta cell function (10). While Arx is necessary to develop into mature alpha cells by repressing the differentiation to both beta and delta cell in pancreatic endocrine differentiation (3). We observed homogeneous expressions of MafA and $P d x 1$ in two insulinomas, and $A r x$ in a glucagonpositive NET. These findings suggested the expression of inherent transcription factors is essential to produce specific hormones in MEN1-associated pNETs, similar to islet endocrine cells. Furthermore, we observed multiple hormone-positive pNET heterogeneously stained with insulin, glucagon and somatostatin. Interestingly, this pNET showed co-expression of MafA, Pdx1 and Arx. From these findings, we assume this sparsely multiple hormonepositive pNET may be functionally immature as pNET, supporting intrinsic transcription factors play key roles in hormone production in MEN1-associated pNETs. It is unclear why this pNET pathophysiologically expressed multiple hormones and transcription factors. However, we speculate that dysgenesis of Ngn3-expressed cells due to menin disruption might also be involved in multiple hormone expression in a single pNET accompanied with functional insufficiency in this MEN1 case.

There are several limitations in this study. First, current study is a single case report. Second, although we demonstrated that transcription factors can play important roles in 'endocrinological maturation' in MEN1- 
associated pNETs, we have not elucidated the relationship between transcription factors and the 'tumorigenesis' in pNETs. In the next step, further studies along with more cases are needed to clarify the underlying mechanisms how pNETs develop and produce intrinsic hormones in MEN1 cases.

In conclusion, our results indicate that transcription factors such as MafA, $P d x 1$ and Arx can play pivotal roles in expression of specific pancreatic hormones in MEN1-associated pNETs, similar to islet endocrine differentiation.

\section{Declaration of interest}

M H received scholarships from Astellas Pharma, Sanofi, Ono Pharmaceutical, Kissei Pharmaceutical, Kyowa Hakko Kirin Co. Ltd., Kowa Pharmaceuticals, Shionogi Inc., Johnson \& Johnson, Daiichi Sankyo, Mitsubishi Tanabe Pharma, Takeda Pharmaceuticals, Taisho Toyama Pharmaceutical Co., Elli Lilly Japan, MSD and Beyer.

\section{Funding}

This research did not receive any specific grant from any funding agency in the public, commercial or not-for-profit sector.

\section{Patient consent}

Written informed consent has been obtained from the patient for this study.

\section{Acknowledgements}

The authors are grateful to the patient who participated in this report.

\section{References}

1 Thakker RV 2014 Multiple endocrine neoplasia type 1 (MEN1) and type 4 (MEN4). Molecular and Cellular Endocrinology 386 2-15. (doi:10.1016/j.mce.2013.08.002)

2 Marx SJ \& Wells SA Multiple endocrine neoplasia. In Williams Textbook of Endocrinology, ed 13, pp 1723-1761. Eds Melmed S, Polonsky KS, Larsen PR \& Kronenberg HM. Publisher is Elsevier, Philadelphia, PA. 2016.

3 Gromada J \& Franklin I 2007 Wollheim CB. $\alpha$-cells of the endocrine pancreas: 35 years of research but the enigma remains. Endocrine Reviews 28 84-116. (doi:10.1210/er.2006-0007)

4 Placzkowski KA, Vella A, Thompson GB, Grant CS, Reading CC, Charboneau JW, Andrews JC, Lloyd RV \& Service FJ 2009 Secular trends in the presentation and management of functioning insulinoma at the Mayo Clinic, 1987-2007. Journal of Clinical Endocrinology and Metabolism 94 1069-1073. (doi:10.1210/jc.2008-2031)

5 Gradwohl G, Dierich A, LeMeur M \& Guillemot F 2000 Neurogenin3 is required for the development of the four endocrine cell lineages of the pancreas. PNAS 97 1607-1611. (doi:10.1073/pnas.97.4.1607)

6 Lee JC, Smith SB, Watada H, Lin J, Scheel D, Wang J, Mirmira RG \& German MS 2001 Regulation of the pancreatic pro-endocrine gene neurogenin3. Diabetes 50 928-936. (doi:10.2337/diabetes.50.5.928)

7 McGrath PS, Watson CL, Ingram C, Helmrath MA \& Wells JM 2015 The basic helix-loop-helix transcription factor NEUROG3 is required for development of the human endocrine pancreas. Diabetes 64 2497-2505. (doi:10.2337/db14-1412)

8 Fontanière S, Duvillié B, Scharfmann R, Carreira C, Wang ZQ $\&$ Zhang CX 2008 Tumour suppressor menin is essential for development of the pancreatic endocrine cells. Journal of Endocrinology 199 287-298. (doi:10.1677/JOE-08-0289)

9 Lu J, Herrera PL, Carreira C, Bonnavion R, Seigne C, Calender A, Bertolino P \& Zhang CX 2010 Alpha cell-specific Men1 ablation triggers the transdifferentiation of glucagon-expressing cells and insulinoma development. Gastroenterology 138 1954-1965. (doi:10.1053/j.gastro.2010.01.046)

10 Cerf ME 2006 Transcription factors regulating beta-cell function. European Journal of Endocrinology 155 671-679. (doi:10.1530/ eje.1.02277)

Received in final form 19 July 2017

Accepted 21 July 2017 